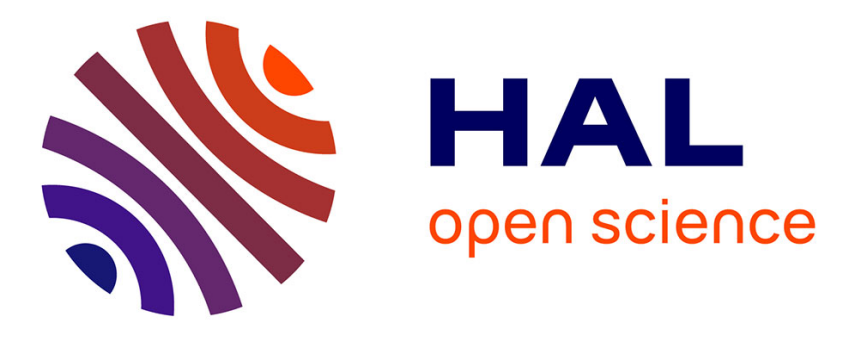

\title{
Estimating the Expected Power Density in a Reverberating Cavity Through Space Averaging
}

Andrea Cozza

\section{To cite this version:}

Andrea Cozza. Estimating the Expected Power Density in a Reverberating Cavity Through Space Averaging. 20th URSI International Symposium on Electromagnetic Theory, Aug 2010, Berlin, Germany. pp.495-498. hal-00511291

\section{HAL Id: hal-00511291 \\ https://hal.science/hal-00511291}

Submitted on 30 Oct 2010

HAL is a multi-disciplinary open access archive for the deposit and dissemination of scientific research documents, whether they are published or not. The documents may come from teaching and research institutions in France or abroad, or from public or private research centers.
L'archive ouverte pluridisciplinaire HAL, est destinée au dépôt et à la diffusion de documents scientifiques de niveau recherche, publiés ou non, émanant des établissements d'enseignement et de recherche français ou étrangers, des laboratoires publics ou privés. 


\title{
Estimating the Expected Power Density in a Reverberating Cavity through Space Averaging
}

\author{
Andrea Cozza \\ Département de Recherche en Électromagnétisme, SUPELEC \\ 3 rue Joliot-Curie, 91192 Gif-sur-Yvette, France \\ andrea.cozza@supelec.fr
}

\begin{abstract}
The use of reverberation chambers often requires the assessment of quantities related to the average power density. This is usually estimated by collecting data from random realizations that are ideally independent, requiring a virtually large number of measurements to be carried out. In this paper, it is proven that an alternative estimator can be defined, requiring just one realization. The estimator is based on a space-averaging approach, and it is shown to be unbiased and asymptotically convergent in probability to the classical ensemble averaging currently used. In other words, power density is shown to be asymptotically ergodic in space. Applications range from faster measurements in reverberation chambers to a solution to the problem of insufficiently large populations of independent samples.
\end{abstract}

\section{INTRODUCTION}

Reverberation chambers are often regarded as environments where statistical field distributions are excited [1] to be used, e.g., for radiated immunity tests in EMC problems. Other applications range from total-radiated-power measurements [2], to the assessment of the electric properties of materials, in particular losses [3]. In all of these configurations, it is necessary to have an accurate estimate of the average power density excited within the chamber. This is usually done by applying in a straightforward way the definition of ensemble average, by generating an ideally large set of statistically independent random samples. The most common way of doing so is to introduce a random variation in the propagation of waves within the chamber, e.g., by modifying the boundary conditions over a region of space [1] (mechanical stirrers), or the propagationrelated phase shift [4] (frequency stirring). Such approach is unfortunately impaired by the limited number of independent samples that can be gathered, so that statistical uncertainty may be inappropriately high [5].

It would thus be beneficial to be capable to compensate such trend by increasing the number of available samples by exploiting space diversity. As the number of degrees of freedom in the chamber increases with its modal density, the spatial correlation of the electromagnetic field is limited to a region of space limited to about one wavelength. It is tempting to think of multiplying the number of samples in space, rather than in the sense required by ensemble statistics. In other words, space ergodicity would help. The aim of this paper is to prove that such property is indeed asymptotically met in an overmoded reverberation chamber. This is done by introducing a discrete plane-wave model in Section II and by introducing an average power density estimator, based on space averaging in a limited region of space; the statistics of this estimator is studied in Section III, where it is proven to converge in probability to the actual ensemble average. Section IV provides some numerical results that show how the estimator accuracy depends on the sample region, thus providing useful information for the design of measurement setups.

\section{DisCRETE PLANE-WAVE SPECTRUM AND POWER DENSITY}

The reverberating cavity will be assumed to be in an overmoded state, i.e., with a high number of resonant modes being significantly excited at the working frequency $f_{0}$. Modal theory [6] states that each resonant mode is associated to a specific field topography, satisfying Helmholtz's equation; by expanding the modal topographies into plane-wave spectra, the field inside an overmoded cavity can be represented as a discrete plane-wave spectrum

$$
\boldsymbol{E}(\boldsymbol{r})=\sum_{p=1}^{N} \gamma_{p} \hat{\xi}_{p} \mathrm{e}^{-\mathrm{j} k_{0} \hat{k}_{p} \cdot \boldsymbol{r}}
$$

where $N$ is the number of plane waves propagating along a phase-congruent path, $\boldsymbol{r}$ is the position at which the electric field is sampled and $k_{0}$ is the propagation constant for the homogeneous medium filling the cavity. The plane-wave spectrum in (1) considers plane waves propagating along the directions $\hat{k}_{p}$, with complex amplitude $\gamma_{p}$ and polarized along the unitary vector $\hat{\xi}_{p}$. The major advantage of this discrete description with respect to the continuous one advocated in [7] is that the discrete one allows highlighting statistical phenomena that are intermediate to the state of perfect reverberation required in [7]. As a matter of fact, a continuous model would lead directly to the results ensured by the central limit theorem, i.e., asymptotic ones. Clearly, the discrete model converges to the continuous one as $N \rightarrow \infty$. Although the expansion proposed in (1) does not allow to appreciate the role of losses in a straightforward way, it is not necessary to assume a lossless configuration.

In the following derivation, the three sets of modal quantities $\left\{\gamma_{p}\right\},\left\{\hat{k}_{p}\right\}$ and $\left\{\hat{\xi}_{p}\right\}$ will be treated as random variables; 


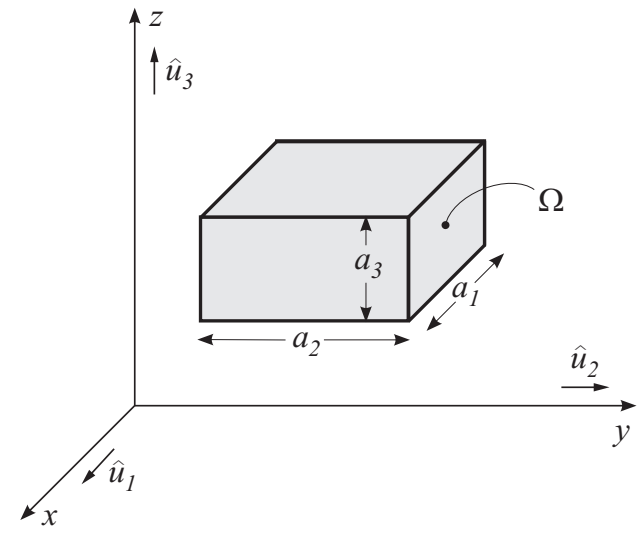

Fig. 1. The sample region $\Omega$ in the shape of a volume.

this should not be regarded as a limitation, but rather as a way of deriving general results that are not constrained by assumptions for a particular configuration of the cavity. The major probabilistic assumptions of this work will be about these three sets: 1) within each set, variables identified by different indexes $p$ will be regarded as independent and identically distributed (iid) and 2) each set will be assumed to be independent from the others. This last assumption is actually non physical, as $\hat{k}_{p} \cdot \hat{\xi}_{p}=0, \forall p \in[1, N]$, although it is not expected to have a major impact on the statistical properties derived in Section III. As reported in several studies [1], [7], the directions of propagation $\left\{\hat{k}_{p}\right\}$ and the polarizations $\left\{\hat{\xi}_{p}\right\}$ can be regarded, for an overmoded cavity, as uniformly distributed over a $4 \pi$ steradian angular region. Conversely, no assumption will be required for the modal weights $\left\{\gamma_{p}\right\}$.

Having introduced our working assumptions, we can now focus on the power density $U(\boldsymbol{r})$. A number of important properties of reverberation chambers are related to this quantity, defined as

$$
U(\boldsymbol{r})=\frac{\|\boldsymbol{E}(\boldsymbol{r})\|^{2}}{\zeta_{0}},
$$

with $\zeta_{0}$ the wave impedance of the filling medium. The expectation of the power density can be defined as

$$
\mu_{U}(\boldsymbol{r})=\mathrm{E}[U(\boldsymbol{r})],
$$

where $E[\cdot]$ stands for the expectation operator (i.e., the ensemble average). It can be easily shown that the average power density is independent of the position $\boldsymbol{r}$, as

$$
\mu_{U}(\boldsymbol{r})=\frac{1}{\zeta_{0}} \sum_{p=1}^{N} \mathrm{E}\left[\left|\gamma_{p}\right|^{2}\right]=\frac{N}{\zeta_{0}} \mathrm{E}\left[\left|\gamma_{p}\right|^{2}\right],
$$

for any $p$, as the $\left\{\gamma_{p}\right\}$ are supposed to be iid. This is usually referred to as the statistical uniformity property of the electromagnetic field in an overmoded cavity [7].

As the computation of $\mu_{U}(\boldsymbol{r})$ implies an ensemble averaging, it implies that an ideally infinite number of independent realizations of modal parameters be considered for a cavity. In EMC applications, this is partly fulfilled by some form of mode stirring; despite of this, the actual number of independent samples is usually limited [5], so that the estimation of the ensemble average is affected by a residual uncertainty. As opposed to this approach, which follows the idea of ensemble averaging, we will prove that an alternative estimator can be defined as

$$
\hat{\mu}_{U}=\frac{1}{M_{\Omega}} \int_{\Omega} U(\boldsymbol{r}) \mathrm{d} \boldsymbol{r}=\langle U(\boldsymbol{r})\rangle_{\Omega},
$$

where $\Omega$ is a region of the cavity defining a volume/surface or stretch of length and

$$
M_{\Omega}=\int_{\Omega} \mathrm{d} \boldsymbol{r}
$$

a measure of the volume/surface/length it occupies. Equation (5) introduces the space-averaging operator $\langle\cdot\rangle_{\Omega}$ as taken over the sample region $\Omega$. With no loss of generality, we will consider that $\Omega$ is a rectangular region with sides $\left\{a_{k}\right\}$ wide, defined along a Cartesian coordinate system, as in Fig. 1. It is important to notice that this estimator has been defined for just one realization of the modal parameters; as it substitutes ensemble averaging with spatial averaging, some kind of spatial ergodicity is understated for power density, a property not yet investigated, to the best of our knowledge.

Substituting (1) into (5), and organizing the modal weights $\left\{\gamma_{p}\right\}$ into the column vector $\gamma$, yields the quadratic form

$$
\hat{\mu}_{U}=\frac{1}{\zeta_{0}} \boldsymbol{\gamma}^{\mathrm{H}} \boldsymbol{P} \boldsymbol{\gamma},
$$

where $\mathrm{H}$ is the Hermitian operator (conjugate transpose) and the elements of matrix $\boldsymbol{P}$

$$
P_{i j}=\left(\hat{\xi}_{i} \cdot \hat{\xi}_{j}^{\star}\right) \phi\left(\hat{k}_{i}-\hat{k}_{j}\right),
$$

where

$$
\begin{aligned}
\phi\left(\hat{k}_{i}-\hat{k}_{j}\right) & =\left\langle\mathrm{e}^{-\mathrm{j} k_{0} \boldsymbol{r} \cdot\left(\hat{k}_{i}-\hat{k}_{j}\right)}\right\rangle_{\Omega} \\
& =\prod_{k=1}^{3} \operatorname{sinc}\left(\pi \frac{a_{k}}{\lambda}\left(\hat{k}_{i}-\hat{k}_{j}\right) \cdot \hat{u}_{k}\right),
\end{aligned}
$$

with $\lambda$ the wavelength for the filling medium and $\hat{u}_{k}$ the Cartesian unit vectors. An important feature of $\boldsymbol{P}$ that is going to be used later on is that the elements on its diagonal are $P_{i i}=1, \forall i \in[1, N]$.

\section{STATISTICS OF THE EXPECTED POWER-DENSITY ESTIMATOR}

In order to assess the accuracy of this estimator for a finite sampling region $\Omega$, the relative error

$$
\epsilon=\frac{\hat{\mu}_{U}-\mu_{U}}{\mu_{U}}
$$

will be considered. The introduction of the random vector

$$
\boldsymbol{v}=\frac{\gamma}{\sqrt{\mathrm{E}\left[\left|\gamma_{p}\right|^{2}\right]}},
$$

which is made up of iid standardized random variables allows recasting (10) in a simpler form

$$
\epsilon=\frac{1}{N} \boldsymbol{v}^{\mathrm{H}} \boldsymbol{P} \boldsymbol{v}-1
$$


In order to be a good estimator, $\hat{\mu}_{U}$ needs to be unbiased, i.e., the following condition needs to be satisfied

$$
\mathrm{E}\left[\hat{\mu}_{U}\right]=\mu_{U} \Longrightarrow \mathrm{E}[\epsilon]=0 .
$$

Before computing $E[\epsilon]$, it is sensible to recall that the independence between the three modal-parameter sets implies the factorization of their joint probability density function (pdf) into

$$
p\left(\left\{v_{p}\right\},\left\{\hat{k}_{p}\right\},\left\{\hat{\xi}_{p}\right\}\right)=p_{\boldsymbol{v}}\left(\left\{v_{p}\right\}\right) p_{\boldsymbol{k}}\left(\left\{\hat{k}_{p}\right\}\right) p_{\boldsymbol{\xi}}\left(\left\{\hat{\xi}_{p}\right\}\right) .
$$

Hence, we can introduce the following notation for conditional averages

$$
\mathbf{E}_{\boldsymbol{v}}[\epsilon]=\int \epsilon\left(\left\{v_{p}\right\},\left\{\hat{k}_{p}\right\},\left\{\hat{\xi}_{p}\right\}\right) p_{\boldsymbol{v}}\left(\left\{v_{p}\right\}\right) \mathrm{d}\left\{v_{p}\right\} .
$$

In this example the standardized modal weights $\left\{v_{p}\right\}$ have been considered, but the same approach can be applied to the other sets. The average in (13) can now be computed by nesting the conditional averages over the three modalparameter sets; starting off with that on the standardized modal weights $\left\{v_{p}\right\}$ at the inner level

$$
\mathrm{E}_{\boldsymbol{v}}[\epsilon]=\frac{1}{N} \mathrm{E}_{\boldsymbol{v}}\left[\boldsymbol{v}^{\mathrm{H}} \boldsymbol{P} \boldsymbol{v}\right]-1 .
$$

Introducing the change of basis $\boldsymbol{P}=\boldsymbol{X} \boldsymbol{\Lambda} \boldsymbol{X}^{-1}$, with $\boldsymbol{\Lambda}=$ $\operatorname{diag}\left\{\lambda_{1}, \ldots, \lambda_{N}\right\}$, and the modal weights vector $\boldsymbol{w}=\boldsymbol{X}^{-1} \boldsymbol{v}$ as expressed over the new basis, we get

$$
\epsilon=\frac{1}{N} \boldsymbol{w}^{\mathrm{H}} \boldsymbol{\Lambda} \boldsymbol{w}-1,
$$

yielding

$$
\mathrm{E}_{\boldsymbol{v}}[\epsilon]=\mathrm{E}_{\boldsymbol{w}}[\epsilon]=\frac{1}{N} \sum_{i=1}^{N} \lambda_{i} \mathrm{E}_{\boldsymbol{w}}\left[\left|w_{i}\right|^{2}\right]-1
$$

as it can be easily proven that the assumption of iid random variables for the set $\boldsymbol{v}$ leads to iid variables for the set $\boldsymbol{w}$, since $\boldsymbol{X}$ is a unitary-norm change of basis. Whence, $\mathbf{E}_{\boldsymbol{w}}\left[\left|w_{i}\right|^{2}\right]=$ $1, \forall i \in[1, N]$, and

$$
\mathrm{E}_{\boldsymbol{w}}[\epsilon]=\frac{1}{N} \sum_{i=1}^{N} \lambda_{i}-1
$$

Since the sum of the $\lambda_{i}$ is equal to $\operatorname{Tr} \boldsymbol{P}$, the trace of $\boldsymbol{P}$, it is not necessary to know the actual eigenvalues. Recalling that $P_{i i}=1, \forall i \in[1, N], \operatorname{Tr} \boldsymbol{P}=N$ and, ultimately

$$
\mathrm{E}_{\boldsymbol{w}}[\epsilon]=0,
$$

which also implies that $\mathrm{E}[\epsilon]=0$ and that the estimator $\hat{\mu}_{U}$ is indeed unbiased. It is remarkable that this result does not depend on the realizations $\left\{\hat{k}_{p}\right\}$ nor $\left\{\hat{\xi}_{p}\right\}$.

Apart being unbiased, $\hat{\mu}_{U}$ needs to be as close as possible to the expected power density $\mu_{U}$. Their distance can be assessed by computing the variance $\sigma_{\epsilon}^{2}$ of $\epsilon$, i.e., its squared rms value. Since $\mathbf{E}[\epsilon]=0$

$$
\sigma_{\epsilon}^{2}=\mathbf{E}\left[\epsilon^{2}\right] \quad .
$$

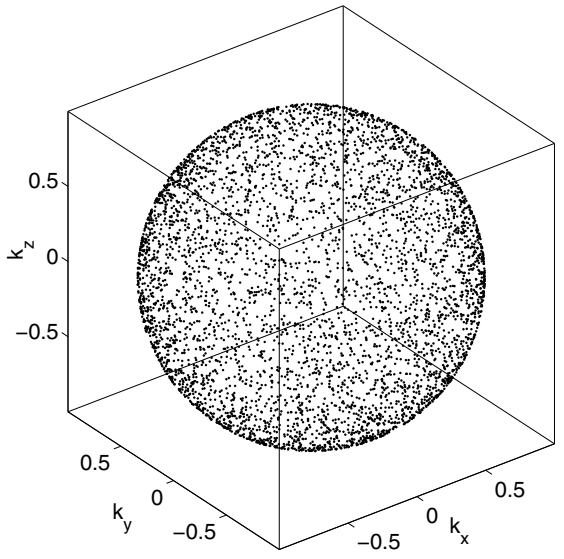

Fig. 2. An example illustrating the assumption of angular uniformity over $4 \pi$ steradians for the modal propagation vectors $\left\{\hat{k}_{p}\right\}$ and the polarization vectors $\left\{\hat{\xi}_{p}\right\}$ : each point represents a realization of a unit vector.

Following the same trail as for the average of $\epsilon$, we consider the nested conditional averages of $\epsilon^{2}$, with the one on the modal weights at the inner level. From (17)

$$
\mathrm{E}_{\boldsymbol{w}}\left[\epsilon^{2}\right]=\frac{1}{N^{2}} \mathbf{E}_{\boldsymbol{w}}\left[\left(\sum_{i=1}^{N} \lambda_{i}\left|w_{i}\right|^{2}\right)^{2}\right]-1
$$

and since

$$
\left(\sum_{i=1}^{N} \lambda_{i}\left|w_{i}\right|^{2}\right)^{2}=\sum_{i=1}^{N} \lambda_{i}^{2}\left|w_{i}\right|^{4}+\sum_{i \neq j} \lambda_{i} \lambda_{j}\left|w_{i}\right|^{2}\left|w_{j}\right|^{2}
$$

we get

$$
\begin{aligned}
\mathrm{E}_{\boldsymbol{w}}\left[\epsilon^{2}\right] & =\frac{1}{N^{2}}\left(\mathrm{E}_{\boldsymbol{w}}\left[\left|w_{i}\right|^{4}\right] \sum_{i=1}^{N} \lambda_{i}^{2}+\right. \\
& \left.+\left(\mathrm{E}_{\boldsymbol{w}}\left[\left|w_{i}\right|^{2}\right]\right)^{2} \sum_{i \neq j} \lambda_{i} \lambda_{j}\right)-1 .
\end{aligned}
$$

The following equivalence can be claimed for the conditional moments $\mathrm{E}_{\boldsymbol{w}}\left[\left|w_{i}\right|^{4}\right]$

$$
\mathrm{E}_{\boldsymbol{w}}\left[\left|w_{i}\right|^{n}\right] \equiv \mathrm{E}\left[\left|w_{i}\right|^{n}\right]=\nu_{n}
$$

as the three modal-parameter sets have been assumed to be independent; the notation $\nu_{n}$ will be used for the sake of simplicity.

A classical result states that

$$
\sum_{i=1}^{N} \lambda_{i}^{2}=\operatorname{Tr}\left(\boldsymbol{P}^{\mathrm{T}} \boldsymbol{P}\right)
$$

and through a short algebraic manipulation, one can show that

$$
\sum_{i \neq j} \lambda_{i} \lambda_{j}=N^{2}-\operatorname{Tr}\left(\boldsymbol{P}^{\mathrm{T}} \boldsymbol{P}\right)
$$


where $\operatorname{Tr}\left(\boldsymbol{P}^{\mathrm{T}} \boldsymbol{P}\right)$ is also related to the Frobenius norm of matrix $\boldsymbol{P}$, here referred to as $\|\boldsymbol{P}\|_{\mathrm{F}}$, by

$$
\operatorname{Tr}\left(\boldsymbol{P}^{\mathrm{T}} \boldsymbol{P}\right)=\|\boldsymbol{P}\|_{\mathrm{F}}^{2}=\sum_{i, j} P_{i j}^{2} .
$$

We can hence state that

$$
\mathbf{E}_{\boldsymbol{w}}\left[\epsilon^{2}\right]=\frac{\|\boldsymbol{P}\|_{\mathrm{F}}^{2}}{N^{2}}\left(\nu_{4}-\nu_{2}^{2}\right) .
$$

Thanks to (28) and (29), we can now express $\sigma_{\epsilon}^{2}$ as

$$
\sigma_{\epsilon}^{2}=\mathrm{E}_{\boldsymbol{\xi}}\left[\mathrm{E}_{\boldsymbol{k}}\left[\|P\|_{\mathrm{F}}^{2}\right]\right] \frac{\nu_{4}-\nu_{2}^{2}}{N^{2}},
$$

where (28) and the independence of $\left\{\hat{k}_{i}\right\}$ and $\left\{\hat{\xi}_{i}\right\}$ yield

$$
\begin{array}{ll} 
& \mathrm{E}_{\boldsymbol{\xi}}\left[\mathrm{E}_{\boldsymbol{k}}\left[\|P\|_{\mathrm{F}}^{2}\right]\right]=N+N(N-1) \cdot \\
\cdot & \mathrm{E}_{\hat{\xi}_{i}, \hat{\xi}_{j}}\left[\left(\hat{\xi}_{i} \cdot \hat{\xi}_{j}^{\star}\right)^{2}\right] \mathrm{E}_{\hat{k}_{i}, \hat{k}_{j}}\left[\phi^{2}\left(\hat{k}_{i}-\hat{k}_{j}\right)\right] .
\end{array}
$$

The correlation term between the polarizations can be shown to be

$$
\mathrm{E}_{\hat{\xi}_{i}, \hat{\xi}_{j}}\left[\left(\hat{\xi}_{i} \cdot \hat{\xi}_{j}^{\star}\right)^{2}\right]=\frac{1}{3} \quad \forall i \neq j .
$$

Whence,

$$
\frac{\sigma_{\epsilon}^{2}}{\nu_{4}-\nu_{2}^{2}}=\frac{1}{N}+\frac{1}{3} \frac{N-1}{N} G(\boldsymbol{a})
$$

with

$$
\begin{aligned}
G(\boldsymbol{a}) & =\mathrm{E}_{\hat{k}_{i}, \hat{k}_{j}}\left[\phi^{2}\left(\hat{k}_{i}-\hat{k}_{j}\right)\right]= \\
& =\iint \phi^{2}\left(\hat{k}_{i}-\hat{k}_{j}\right) p_{\hat{k}}\left(\hat{k}_{i}\right) p_{\hat{k}}\left(\hat{k}_{j}\right) \mathrm{d} \hat{k}_{i} \mathrm{~d} \hat{k}_{j}
\end{aligned}
$$

the only term dependent on the physical extension of $\Omega$. Column vector $\boldsymbol{a}$ gathers the widths $\left\{a_{k}\right\}$ of $\Omega$, whereas $p_{\hat{k}}(\hat{k})$ is the pdf associated to the directions of propagation.

Thanks to Tchebychev inequality, this result proves that $\hat{\mu}_{U}$ converges in probability towards the actual expectation of the power density as soon as $G(\boldsymbol{a})$ goes to zeros. Otherwise, the estimator will always be affected by a finite residual uncertainty, even for a large number of plane waves propagating inside the cavity.

\section{NumericAl RESUlts}

As the accuracy of $\hat{\mu}_{U}$ is dominated by $G(\boldsymbol{a})$, its dependence from the geometrical dimensions of $\Omega$ is here considered. To this end, (34) has been evaluated numerically, through a Monte Carlo approach, considering the generic $\hat{k}_{p}$ as distributed according to the following pdf

$$
p_{\hat{k}}(\vartheta, \varphi)=\frac{\sin \vartheta}{4 \pi},
$$

where $(\vartheta, \varphi)$ are the elevation and azimuthal angles of a spherical coordinate system. This leads to the uniform distribution shown in Fig. 2. Two sets of random directions were computed in this way, leading to the ensemble average in (34). Three cases were analyzed, by considering a varying number of nonnull dimensions $a_{k}$ in $\Omega$. For the sake of simplicity, all of these

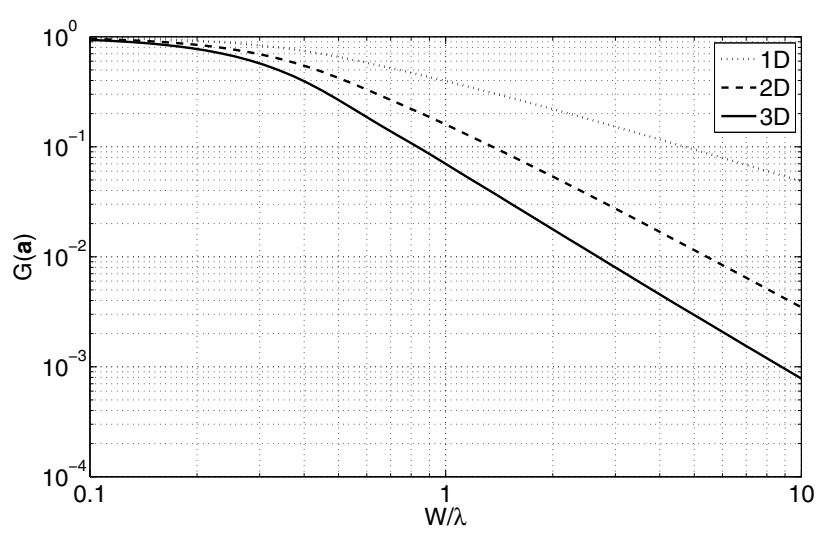

Fig. 3. Results from the Monte Carlo integration of (34). For the three cases considered, the non-null dimensions have been set equal to $W$.

were imposed to be equal to $W$, leading to a cubic/square or linear region of space. The results thus obtained are shown in Fig. 3: as expected, an increasingly larger sample region $\Omega$ ensures a lower residual error of the estimator. In particular, as soon as $W \gtrsim \lambda, \sigma_{\epsilon}^{2}=\mathcal{O}\left((W / \lambda)^{-1}\right)$ for a one-dimensional $\Omega$ and $\sigma_{\epsilon}^{2}=\mathcal{O}\left((W / \lambda)^{-2}\right)$ for a three-dimensional one, with the two-dimensional configuration in between. Hence, e.g., an rms relative error $\sigma_{\epsilon} \simeq 10 \%$ can be attained by sampling the electric field over a square region about $5 \lambda$ wide.

\section{CONCLUSIONS}

We have proven that space ergodicity is asymptotically met in overmoded reverberation chambers. The rate of statistical convergence is dictated by the number of degrees of freedom (plane waves) but a lower bound in the accuracy is enforced by the electrical dimensions of the region over which the field is being measured. Numerical results show that relatively small regions allow to attain acceptably low statistical uncertainties, thus providing the means to improve the performance of applications requiring an estimation of the average power density.

\section{REFERENCES}

[1] P. Corona, G. Latmiral, and E. Paolini, "Performance and analysis of a reverberating enclosure with variable geometry," IEEE Trans. on Electromagn. Compat., pp. 2-5, 1980.

[2] P. Corona, G. Latmiral, E. Paolini, and L. Piccioli, "Use of a reverberating enclosure for measurements of radiated power in the microwave range," IEEE Trans. on Electromagn. Compat., pp. 54-59, 1976.

[3] D. Hill, M. Ma, A. Ondrejka, B. Riddle, M. Crawford, and R. Johnk, "Aperture excitation of electrically large, lossy cavities," IEEE Trans. on Electromagn. Compat., vol. 36, no. 3, pp. 169-178, 1994.

[4] D. Hill, "Electronic mode stirring for reverberation chambers," IEEE Trans. on Electromagn. Compat., vol. 36, no. 4, pp. 294-299, 1994.

[5] K. Madsen, P. Hallbjorner, and C. Orlenius, "Models for the number of independent samples in reverberation chamber measurements with mechanical, frequency, and combined stirring," IEEE Antennas Wireless Propag. Lett., vol. 3, 2004.

[6] J. Van Bladel, Electromagnetic fields. IEEE Press Series on Electromagnetic Wave Theory, 2007.

[7] D. Hill, "Plane wave integral representation for fields in reverberation chambers," IEEE Trans. on Electromagn. Compat., vol. 40, no. 3, pp. 209-217, 1998. 